\title{
La representación de la muerte en el videojuego ${ }^{1}$ The representation of death in video games
}

\author{
Diego Maté2
}

\begin{abstract}
Resumen
Desde sus inicios, el videojuego desplegó un espectro estético que posibilitó nuevas formas de representar la muerte. Podría pensarse que las diferencias entre el videojuego y otros medios como el cine surgen del encuentro de dos grandes formas culturales como el relato y el juego, pero el panorama actual muestra un escenario pleno de mixturas y pasajes. En este trabajo se señalan los rasgos característicos que adoptó la representación de la muerte en el videojuego como la recurrencia, su inserción en un régimen lúdico, su distanciamiento de las semantizaciones más frecuentes propias de géneros narrativos y su tendencia a la disrupción del relato. Además, se describen dos grandes estrategias representativas que el medio elaboró en distintos momentos de su historia, y que en la actualidad conviven y dominan en buena medida el paisaje estético videolúdico: en una se sugiere el acto de morir invisibilizándolo, en otra se apuesta a la espectacularización de la muerte a través de una maximización audiovisual. Estas dos formas no se presentan en estado de pureza ni excluyen otras vías representativas posibles, pero permiten observar tendencias que gozaron (y gozan) de una gran estabilidad. Por último, se analizan cuatro casos que producen un desvío respecto de la implementación del acto de morir como penalización y retroceso de carácter lúdico, y proponen soluciones alternativas a la cuestión en torno de la representación de la muerte.
\end{abstract}

Palabras clave: Videojuego; muerte; representación; sociosemiótica

\section{Abstract}

From its beginnings, videogames deployed an esthetic spectrum that made new forms of representing death possible. It could be thought that differences between videogame and other media, such as cinema, arise from the clash between two great cultural forms: storytelling and gaming. But current context shows a scenario that is full of mixtures and connections between them. In this work, characteristic features of death representation in videogames -such as recurrence, insertion in a playful regime, distance from typical semantizations of narrative genres, and tendency to disrupt the story- are pointed out. In addition, this work describes two great representative strategies that the medium elaborated in different moments of its history. At present, both of them coexist and to a great extent dominate the video-ludic landscape. One strategy consists in invisibilizing the act of dying; in the other one, death is spectacularized through an audiovisual maximization. These two forms do not present themselves in a pure state nor do they exclude other possible representative paths, but they allow us to observe tendencies that enjoyed (and enjoy) a great stability. Finally, four cases are analyzed. Such cases produce a deviation from the implementation of the act of dying as a penalty and as a regression of ludic nature, and propose alternative solutions to the question about the representation of death.

Keywords: Video game; death; representation; social semiotic

1. Una primera versión de este trabajo fue presentada con el título "Videojuego: Norma y desvío en la representación de la muerte y sus efectos" en CIVE III (Tercer Congreso Internacional de Videojuego y Educación), realizado el 12,13 y 14 de agosto de 2015 en Buenos Aires. A su vez, el texto continúa las indagaciones abiertas por otro artículo, "Representaciones de la muerte en el videojuego. Lo lúdico y lo narrativo entre la norma y el desvío", publicado en la revista Lexia. Rivista di semiotica, en 2016.

2. Licenciado en Crítica de Artes. Instituto de Investigación y Experimentación en Artes y Crítica (IIEAC), Área Transdepartamental de Crítica de Artes, Universidad de las Artes. Argentina. Correo electrónico: diegomateyo@gmail.com. ORCID ID: 0000-0001-5791-0847 
Tipología: Artículo de reflexión

Recibido: $18 / 08 / 2017$

Evaluado: $30 / 10 / 2017$

Aceptado: $16 / 11 / 2017$

Disponible en línea: 29/12/2017

Como citar este artículo: Maté, D. (2018). La representación de la muerte en el videojuego. Jangwa Pana, 17 (1), 61-71. Doi: http://dx.doi.org/10.21676/16574923.2296

\section{Introducción}

a cuestión de la representación de la muerte Len el videojuego se inscribe en el espacio de una discusión decisiva en los game studies, campo que emerge a finales de los 90 con el incremento de investigaciones y obras dedicadas al tema, además de la fundación de la revista especializada Game Studies. En ese momento inicial se perfilan dos posturas, la de la ludología y la narratología, que habrían de modelar el campo en torno a una polémica sobre el destino del medio y de las investigaciones futuras. De un lado, los ludólogos, como Espen Aarseth, Jesper Juul, Gonzalo Frasca y Aki Järvinen, reclamaban la constitución de un espacio académico con preocupaciones, conceptos y métodos de análisis originales que partieran de las indagaciones dispersas en relación con el juego hechas por autores como Johan Huizinga, Roger Caillois, Bernard Suits y Brian Sutton-Smith. La ludología rechazaba la utilización del herramental de otras disciplinas como el análisis fílmico o narrativo por considerar que el videojuego, en tanto forma lúdica, requería de un abordaje singular. La corriente rival incluyó a autores como Janet Murray y Henry Jenkins que mostraban interés por las posibilidades narrativas del videojuego y su inserción en la escena de los nuevos medios de comunicación. La discusión quedó servida tempranamente en 1997, cuando se publicaron los libros de Aarseth y Murray ${ }^{3}$ que habrían

3. Se trata de Cybertext: Perspectives on ergodic literature (Aarseth 1997) y Hamlet on the Holodeck: The future of narrative in Cyberspace (Murray 1997). de anticipar el rumbo del intercambio los años siguientes.

Más allá de la exageración de algunas posiciones, del tono prescriptivo de muchos planteos y de la virulencia del debate ${ }^{4}$, la polémica habría de desdibujarse a medida que el campo creciera en volumen y diversidad de perspectivas. En 2005, Jesper Juul, inicialmente enrolado en la postura ludológica, publica Half-Real donde se propone al medio como un artefacto atravesado tanto por elementos del orden del juego como ficcionales.

Esa disputa académica puso de relieve una tensión entre lo lúdico y lo narrativo que engloba tanto las indagaciones realizadas alrededor del medio como su producción discursiva. El tema resulta demasiado extenso como para abordarlo aquí, pero la cuestión puede reducirse a los distintos funcionamientos semióticos que suponen dos formas culturales como el relato y el juego. Durante mucho tiempo, hasta el siglo XX, una $\mathrm{y}$ otra forma se asentaron en espacios sociales más o menos circunscriptos y separados, pero el surgimiento del videojuego en los 70 y sus intentos de apropiarse de modalidades narrativas de otros medios (como el cine y la literatura) dieron lugar a cruces múltiples y originales entre juegos y relatos. La cuestión de la representación de la muerte en el videojuego abordada a continuación se inscribe en el terreno abierto por esa tensión específica del medio.

4. Frasca, que intervino en la discusión, tiempo después relativizó las diferencias entre las dos posturas (Frasca 2003). 
Desde una perspectiva sociosemiótica, en este trabajo se presentan distintas modalidades del fenómeno y se analizan cuatro casos que ponen en práctica desvíos particulares. El análisis toma como eje la representación de la muerte atendiendo a su vinculación con las dimensiones lúdica y narrativa, y se realiza teniendo en cuenta tres niveles: retórico, temático y enunciativo (Steimberg, 2013). El desarrollo apunta a describir el funcionamiento de la representación de la muerte en términos de producción de sentido y de la generación de una propuesta de vínculo discursiva (Verón, 1996). Se trata de evidenciar cómo el medio (re)trabaja un motivo y/o tema (Segre, 1988) plenamente vigente y de larga vida histórica y, al mismo tiempo, observar cómo el acto de morir es reelaborado tanto al nivel de la representación como del devenir lúdico, es decir, cómo se plasma la muerte en la superficie discursiva, mediante qué figuraciones, pero también cómo se articula ese despliegue audiovisual con el régimen lúdico específico del videojuego; por ejemplo, cómo entra en relación con el nivel de las reglas y los objetivos del juego, impone restricciones, permite reinvenciones temáticas o habilita nuevos contratos enunciativos.

\section{Discusión}

\section{La muerte lúdica}

El rasgo más característico de la muerte en el videojuego es la recurrencia. En el videojuego es normal que el avatar (personaje o entidad controlado por el jugador) muera; el hecho de morir con frecuencia no produce ningún tipo de disrupción como sucedería, por ejemplo, dentro del marco de un relato, debido a que esa recurrencia forma parte de las previsibilidades del medio. Esto está relacionado con la temporalidad del videojuego que tiende hacia la repetición y la reelaboración de las acciones. Al no presentar una progresión cronológica lineal, el videojuego diseña estructuras temporales cuya textura no se parece a la de los géneros narrativos asentados en otros medios. La muerte, recurrente y previsible, es uno de los pivotes de esa temporalidad que pone en entredicho no solo las concepciones de tiempo y linealidad, sino también la de memoria (Mukehrjee, 2009).

Esa recurrencia ha sido objeto de críticas y cuestionamientos. Algunos autores, como Gonzalo Frasca, sostuvieron que el videojuego trivializa la muerte y el "valor sagrado de la vida" al hacer del morir algo reversible y nunca final (citado en Mukehrjee, 2009). Sin embargo, también podría argumentarse que el medio abre el camino para una representación nueva de la muerte que se distancia de lo hecho por otros medios. Por ejemplo, la muerte cumple una función central dentro del marco de la textualidad lúdica del videojuego: es una manera de conocer las reglas del juego, de poner a prueba su sistema, de aprender los usos correctos y los incorrectos hasta conseguir un cierto nivel de experticia. En géneros que construyen una escena enunciativa particularmente centrada en el despliegue de aptitudes físicas y cognitivas, como el de plataformas, de lucha callejera o el first person shooter, la muerte instaura un régimen de aprendizaje en donde la penalidad y el retroceso se transforman en las herramientas privilegiadas de una pedagogía que se construye sobre la corrección gradual de los propios errores (Neto, 2015). Tocci (2008) explica que la dinámica de prueba y error interrumpe la progresión narrativa, obligando al jugador a enfocarse más tiempo en el sistema de reglas que en la ficción, y pone de relieve así una tensión propia del medio entre las dimensiones lúdica y de la narración.

Juul, por su parte, cree que la muerte en el videojuego pone de manifiesto un fenómeno social que denomina paradoja del fracaso, y que puede resumirse en las siguientes premisas: "1. Generalmente evitamos el fracaso, 2. Experimentamos el fracaso cuando jugamos juegos, 3. Buscamos juegos, a pesar de que experimentaremos algo que normalmente evitamos" (Juul, 2013. p. 2). El autor sos- 
tiene que la fascinación que producen los juegos (no solo los videojuegos) surge del hecho de que revelan a sus participantes que se carece de habilidades para cumplir exitosamente ciertas tareas, al mismo tiempo que posibilitan la adquisición y mejoramiento de esas habilidades.

Otro rasgo es el componente lúdico que adopta la muerte cuando es representada en el videojuego. Lejos de las tematizaciones narrativas más frecuentes, en el videojuego es común que el acto de morir se vuelva un "significante del fracaso" (Harrer, 2013). La muerte lúdica, al menos en un principio, no tiene obligación de referir más que a un uso incorrecto del juego, es decir, al fracaso en el intento de superar los objetivos. En la actualidad, con juegos que se apoyan en estructuras cada vez más narrativas que imitan al cine y a la literatura, esto cambió: la muerte puede aparecer revestida de ciertas características semánticas habituales en otros medios como el sacrificio, la expiación, el duelo, etc. Esos motivos, sin embargo, son relativamente nuevos en la historia del videojuego, y durante mucho tiempo los juegos no necesitaron replicar las semantizaciones de los géneros narrativos de otros medios, ya que la muerte cumplía una función lúdica y señalaba una penalización de la performance.

El tercer rasgo consiste en que la muerte lúdica, además de dificultar la organización de un relato, obliga a integrarlo al régimen lúdico. $\mathrm{Al}$ ser el acto de morir recurrente y previsible, la narración de un juego debe trabajar con esa reversibilidad y ajustarse a una convención histórica del medio. Así, no es extraño que durante mucho tiempo, más o menos hasta la llegada de los motores gráficos en tres dimensiones durante los 90, al videojuego le resultara casi imposible desplegar relatos que imitaran los modos constructivos del cine ${ }^{5}$. No fue

5. En relación con el concepto de narración, se trabaja a partir de estudios de Roland Barthes (1972) y Christian Metz (1991), donde se entiende lo narrativo como una instancia vincular, de carácter metatextual y que hasta que se pudo comenzar a imitar con cierto nivel de detalle la gramática cinematográfica tradicional (con su escalaje en planos, el recurso del plano contraplano, las escenas de diálogo, la utilización del montaje para construir el ritmo, etc. ${ }^{6}$ ) que el videojuego comenzó a apostar a narraciones cada vez más complejas. Sin embargo, la reversibilidad de la muerte supone un obstáculo: la recurrencia del morir expulsa al jugador de la trama y le recuerda constantemente la naturaleza lúdica del juego; el fluir de los personajes y sus conflictos cede ante el funcionamiento de las reglas y los objetivos a cumplir, volviendo la inmersión narrativa mucho más difícil.

\section{Dos estrategias representativas}

Si bien los rasgos analizados son característicos de la representación que suele elaborar el videojuego, a lo largo de los años esos rasgos dieron lugar a configuraciones más o menos estables que llamaremos estrategias. La primera estrategia surge con el videojuego mismo y consiste en una representación ambigua que sugiere la muerte sin hacerla visible, acentuando el señalamiento del fracaso y la penalización antes que la muerte en sí misma. Desde los primeros juegos de comienzos de los 70 hasta el presente, esta estrategia supuso una manera inédita de representar la muerte, inhallable en otros medios. Aquí la muerte se sugiere mediante ciertas figuraciones y transformaciones del cuerpo del avatar, pero sin poner en escena el acto en cuestión. Mencionaremos solo algunos ejemplos vinculados a géneros específicos. En los juegos de lucha callejera (o beat'em up), como Double Dragon (1987) o Final Fight (1989), el avatar derrotado, después de haber sufrido graves heridas físicas y de haber agotado su barra de energía, cae al suelo y titila

puede tomar a su cargo la totalidad de operaciones del discurso.

6. Se tiene en cuenta aquí el conjunto de convenciones y desarrollos fílmicos identificados por Noel Burch (2011) como Modo de Representación Institucional, y que llegan hasta el presente. 
hasta que desaparece y otra instanciación del mismo avatar se materializa. En los juegos de plataformas, como Super Mario Bros (1985) o Wonderboy (1986), cuando el avatar colisiona contra un enemigo u obstáculo, se eleva y cae hasta salir de los límites de la pantalla mientras observa hacia la pantalla con cara de incomodidad. En ambos casos, se pierde una vida del contador; esta pérdida, sumada al titilar/salto y desaparición posterior, configura una muerte que no deja ver sus signos más convencionales como un cuerpo destrozado, heridas o sufrimiento. La muerte, en todo caso, surge como efecto de sentido (Verón, 1996) producto de las operaciones del texto que la sugieren: un jugador podrá decir que el protagonista murió, aunque la elaboración realizada por el discurso aluda a una situación que oscila entre el morir y el fracaso lúdico. Se trata de una muerte desplazada, que se sugiere a partir de convenciones como el titilar o el saltar mirando en dirección a la pantalla y por el hecho de restarse una vida del contador y la desaparición momentánea del avatar.

Con el avance tecnológico, el videojuego pudo apropiarse de algunas convenciones audiovisuales de otros medios como el cine o la televisión. La representación de la muerte cambió sobre todo a partir de Mortal Kombat (1992), que inauguró un régimen representativo nuevo dentro del videojuego. A diferencia de los casos descritos, en Mortal Kombat, un juego de lucha, la muerte comenzó a representarse con una crudeza tal que la franquicia se hizo famosa por la violencia que el ganador del combate descargaba sobre el cuerpo del rival derrotado. En la primer entrega de la serie, cada luchador puede realizar una fatality que requiere una combinación de comandos específica: la pelea no termina con la victoria de un personaje sobre el otro, sino con un vistoso movimiento final en el que la representación de la muerte suspende el devenir lúdico.
Una vez ejecutada correctamente la fatality, la acción se detiene, los controles son interrumpidos y lo que queda es observar una secuencia de acciones en la que el vencedor desmiembra, incendia o electrocuta a un rival incapacitado. Con cada nuevo juego, la franquicia incrementa el número de luchadores así como de fatalities y el despliegue audiovisual de las mismas, cada vez más cinematográfico.

De Mortal Kombat en adelante, muchos juegos experimentaron con este nuevo régimen representativo. La saga de Resident Evil (1996-2017), por ejemplo, escenifica el fracaso del jugador mediante cinemáticas (Klevjer, 2002) en las que se representa la muerte recuperando elementos del lenguaje del cine de terror y gore. En Skyrim (2011), la última entrega de la serie The Elder Scrolls, cuando el avatar es derrotado, se ralentiza la acción y cambia el ángulo de visión con el fin de maximizar la visualidad: la muerte, que puede producirse de incontables maneras, es mostrada en cámara lenta. En este nuevo régimen, la representación de la muerte se vuelve un espectáculo coreografiado que los juegos hiperbolizan al punto de detener el flujo de la interacción para ofrecer al jugador un acceso privilegiado a los hechos: el acto de morir deviene un show que se adueña de la pantalla.

Cabe preguntarse qué es lo que esta nueva forma de representación obtura. Así como se dijo que la primera estrategia elidía la representación de la muerte y la sugería a través de ciertas convenciones (como el titilar y desaparecer), esta segunda estrategia acentúa la espectacularización mientras clausura cualquier tipo de empatía con el cuerpo sufriente: lo que escapa a la representación es el dolor del personaje que ocupa el lugar de víctima sacrificial. Megan Blythe Adams (2014) sostiene que "esta clase de muerte del jugador es visualmente suntuosa, expresando la frustración (incluso la agonía) de la muerte mediante algo que asemeja una danza ritual”. 
Estas dos estrategias parecen haber dominado la representación de la muerte en el videojuego. Al día de hoy, una y otra continúan señalando los límites expresivos del medio a la hora de tematizar el acto de morir: las dos formas devinieron norma y redujeron el espectro de lo decible (Metz, 1975) del videojuego. A continuación, se analizarán algunos casos que toman distancia de esos verosímiles y que abren otros espectros representativos y temáticos posibles. Estos casos serán presentados conformando un gradiente que va desde un pequeño desvío a una reformulación total.

\section{Karoshi}

La serie Karoshi cuenta con seis juegos; todos replican la iconografía y la espacialidad de los juegos de plataformas, pero se decantan por una dinámica de juego de ingenio que difiere de aquel en relación con los objetivos y las competencias con las que se recorta al enunciatario: mientras que en el juego de plataformas el jugador debe sortear grandes porciones de terreno para llegar a un destino, en los juegos de ingenio (puzzle) se trata de resolver problemas que se presentan bajo la forma de un enigma lógico. En el caso de Karoshi, el desafío consiste en lograr que el protagonista alcance a suicidarse realizando una secuencia de acciones concretas. El señor Karoshi, un empleado de oficina, debe provocar su propia muerte empleando de manera óptima los elementos con que lo provee cada nivel ${ }^{7}$. La muerte puede producirse por aplastamiento, caída sobre picos filosos, electrocutamiento o por un disparo, entre otras causas. La dinámica lúdica no es distinta a la de muchos juegos puzzle que toman elementos del género de plataformas, como Lemmings (1991) o Krustys Fun House (1992); la novedad reside en que aquí el objetivo (la solución de cada problema) no consiste en hallar la salida

7. En japonés, karoshi significa "muerte por exceso de trabajo" (Gorvett, 2016). de un laberinto, sino en lograr encontrar los medios indicados para que el tragicómico señor Karoshi pueda suicidarse, un nivel tras otro. El juego trastoca el universo habitual puzzle mientras que deja intacta su estructura lúdica. La dinámica de solución de problemas lógicos es fiel a la vertiente más tradicional del género, el desvío se produce en un plano temático: la sátira de la serie se asienta en el hecho de que la solución de cada nivel suponga encontrar la vía adecuada para el suicidio.

\section{This War of Mine}

This War of Mine (2014) evoca libremente el asedio de Sarajevo durante la guerra bosnia. El juego pertenece a un género reciente, el de supervivencia. El jugador controla inicialmente a tres personajes que habitan una casa desvencijada durante el bombardeo de la ciudad. El juego se divide en dos momentos bien diferenciados: el día, cuando los personajes deben realizar labores de todo tipo aprovechando el tiempo de la manera más eficiente posible (cocinar, alimentarse, dormir, tapiar ventas, efectuar arreglos, fabricar instrumentos -como armas o herramientas-, divertirse, negociar con mercaderes, acudir en la ayuda de vecinos en problemas, etc). En cambio, durante la noche se debe elegir un personaje y hacer que se dirija a algunas de las zonas de la ciudad para proveer al grupo de materiales, comida, medicamentos y demás insumos: con ese fin, puede saquear, robar y matar a los ocupantes de los lugares a visitar (casas de familia, edificios abandonados, destacamentos militares, hospitales). El objetivo principal del juego es sobrevivir, y para conseguirlo se necesita alcanzar un nivel satisfactorio de autosustentación: eventualmente, cuando el asedio recrudece y la cantidad de lugares disponibles disminuye, los personajes deben ser capaces de cultivar sus alimentos, preparar sus medicinas, fabricar sus municiones, filtrar el agua de lluvia, etc. 
El género de supervivencia, que puede incluir elementos de otro género, el de administración, se encuentra en expansión y, salvo por el hecho de estar inspirado en el asedio de Sarajevo, This War of Mine exhibe semejanzas con Terraria (2011), Minecraft (2011), Rust (2013) y Don't Starve (2013). El género produce una torsión en la representación habitual de la muerte: mientras que en la gran mayoría de los juegos esta es reversible y no supone un estado final, en los juegos de supervivencia es permanente. Si un personaje de This War of Mine muere durante una de sus excursiones nocturnas, o si lo hace por una enfermedad o se suicida en la casa, el jugador es despojado de ese avatar de manera permanente y debe intentar lograr la autosustentación del grupo habiendo perdido a uno de sus integrantes, lo que complica la gestión eficaz del escaso tiempo disponible y la realización de las tareas cotidianas, además de las excursiones nocturnas de abastecimiento.

This War of Mine aborda lo que casi todos los juegos eliden: los efectos de la muerte en los otros (Maté, 2016). Cada personaje posee una serie de rasgos físicos y psicológicos que lo vuelve único y una pieza decisiva dentro de la precaria economía de la casa. La mayoría, por ejemplo, reacciona experimentando culpa y remordimiento después de haber cometido un asesinato en una expedición. Si el personaje vuelve a matar, eso puede sumirlo en una profunda depresión que lo torna ineficiente durante el día, y si no se encuentra la manera de levantarle el ánimo (por ejemplo, haciendo que alguno de sus compañeros dedique un tiempo a consolarlo), el personaje puede llegar al suicidio. A su vez, la muerte de un integrante de la casa afecta de distintas maneras a sus compañeros: la mayoría se verá conmovida con su ausencia, mientras que algunos pueden deprimirse o quebrarse y llorar imprevistamente, interrumpiendo sus tareas cotidianas. Esta mella en el temple del grupo puede durar varios días; el malestar puede leerse a través de los globos de diálogo en los que un personaje puede llegar a decir varias veces cosas como "no puedo creer que $\mathrm{X}$ ya no esté con nosotros".

Salvo por algún otro caso inusual, como The Sims, ese tratamiento de la muerte de This War of Mine es prácticamente inédito en la historia del videojuego: quizás por primera vez, la muerte produce inevitablemente alguna clase de reacción en los personajes: la forma que adopte esa reacción dependerá de los rasgos que compongan la psicología de cada avatar. This War of Mine suma una dimensión humana al acto de representar la muerte, exhibe el dolor en los otros, la culpa, el arrepentimiento, el recuerdo. Pero no se trata solo de un quiebre exclusivamente temático ya que, como se dijo antes, esos efectos pueden llegar a influir enormemente en el desarrollo de la partida: la depresión de un asesino, por ejemplo, puede dejarlo postrado en la cama, haciendo que se cuente con un personaje menos para completar las tareas cotidianas necesarias para garantizar la supervivencia del grupo. Así, la representación novedosa de la muerte y de sus secuelas en la mente y el cuerpo tiene su correlato en la dimensión lúdica: los efectos psicológicos negativos se traducen en obstáculos para la gestión óptima de la casa.

\section{Every Day the Same Dream}

En el juego del estudio italiano Molleindustria (2009) se controla a un protagonista que debe ir a trabajar a su oficina en la mañana. En el ascensor de su edificio, el avatar se cruza con una anciana que le dice: "Cinco pasos más y tu vida cambiará". Esos "pasos" no son otra cosa que elementos con los que el personaje debe interactuar, acciones a realizar en su rutina matinal camino al trabajo: hablarle a un hombre sin techo, recoger y observar una hoja de árbol que cae, acariciar una vaca, etc. El recorrido del personaje es lineal, de la casa a la oficina, y si el jugador no interactúa 
con esos elementos, ese día-sueño puede acabar de dos maneras: con el avatar sentándose a trabajar en su cubículo o arrojándose del edificio de la empresa. Una u otra alternativa dan por igual inicio a un nuevo día-sueño que trae una nueva oportunidad para encontrar los "pasos" (o, podría pensarse, también: es el mismo sueño que recomienza). Una vez que los cinco elementos son completados, el avatar despierta igual que todos los días, pero el mundo parece deshabitado: faltan su esposa, la anciana, los autos, su jefe y sus compañeros. Cuando llega a la terraza del edificio, se ve a sí mismo arrojándose desde el techo: el juego no esclarece el sentido de esa escena, pero da a entender que esa visión indica un cambio en el protagonista producto de ciertas desobediencias (como ir al trabajo en calzoncillos) y de haber dirigido su atención a las pequeñas cosas de su vida cotidiana (como una vaca, a la que acaricia después de haberse bajado de su auto en medio de una autopista atestada de vehículos).

La singular representación de la muerte de Every Day the Same Dream consiste en ese tratamiento onírico que el título del juego anuncia: si el jugador no logra advertir los elementos con los que se debe interactuar para completar los "pasos", como lo explica misteriosamente la anciana del ascensor, el recorrido lineal hacia la oficina puede finalizar, como ya se dijo, de dos maneras: una de ellas es el arrojarse al vacío desde un edificio. Ese suicidio no está presentado como una acción realizada en el mundo real, sino en otra clase de entorno, quizás en un sueño, donde la muerte no solo no se representa (no se ve, por ejemplo, el resultado de la caída, el cuerpo en el pavimento), sino que tampoco supone ninguna clase de penalidad lúdica ni de conflicto narrativo: el día-sueño comienza de nuevo, el jugador puede no detectar los pasos restantes y arrojarse del edificio un número infinito de veces. Finalmente, la visión del protagonista, cuando se ve a sí mismo tirándose del balcón, confirma el carácter onírico de la historia y su desvío representativo de las estrategias antes men- cionadas: aquí el acto de morir no está asociado al fracaso sino, por el contrario, a alguna clase de emancipación, pero se trata de una emancipación ambigua que se presenta con cierta indeterminación, por lo que la muerte aparece asociada a lo onírico y se despliega en un terreno indecidible: las escenas que el juego ofrece, ¿pertenecen al ámbito de la realidad, del sueño o a alguna especie de fantasía? No se sabe, Every Day the Same Dream toma un partido representativo distinto que opta por la ambigüedad y por un tratamiento inédito de la muerte, alejado en buena medida del régimen lúdico (los objetivos son débiles, ceden ante el clima de incertidumbre general) tanto como de las estrategias figurativas más frecuentes. Aquí la muerte es tanto ensoñación como una suerte de vía para el autoconocimiento.

\section{The Graveyard}

Los casos analizados hasta ahora pueden ser ubicados en un gradiente: del cambio únicamente representacional que operaban los juegos de Karoshi, pasando por la novedad de abordar los efectos y las secuelas de la muerte en los otros (This War of Mine), llegando hasta la modificación del espectro temático de la muerte al fundirlo con una lógica onírica y contemplativa (Every Day the Same Dream). El caso de The Graveyard (2008) es distinto y marca los límites borrosos de una frontera, la que separa lo lúdico de otro registro discursivo. Ya Everyday the Same Dream daba cuenta de una notable disminución del espesor del nivel lúdico: si bien el juego puede ser entendido como una serie de puzzles ligeros, de corta duración y con desafíos relativamente sencillos, se verifica una primacía del trabajo con el clima y con lo audiovisual por sobre la dinámica de juego. Los objetivos no organizan de manera rígida la exploración, que puede realizarse con cierto grado de libertad sin que la muerte funcione como amenaza. The Graveyard, por su parte, carece de objetivos; eso lo coloca enseguida dentro de una clase de textos distintos 
al del videojuego; un tipo de texto igualmente ergódico, ya que se demanda la participación activa de un usuario para que el texto pueda desenvolverse (Aarseth, 1997), pero no lúdico, ya que no posee una estructura compuesta de reglas y objetivos $\mathrm{y}$, por eso mismo, no genera una escena enunciativa como la del videojuego, en la que se recorta a un enunciatario a través de ciertas competencias de diversos tipos (cognitivas, de reacción, lógicas, etc.).

El juego del estudio Tale of Tales consiste en la siguiente escena: el jugador controla a una anciana que cruza un cementerio por un camino recto. El personaje se mueve hacia adelante hasta llegar a un banco junto a una pared. Allí el personaje solo puede sentarse y, en ese punto, se pierde el control del avatar. Inmediatamente, por encima de la imagen de la anciana sentada, se sobreimprimen imágenes de ella en primer plano mientras de fondo comienza a escucharse una canción folklórica en alemán que habla de personas fallecidas (presumiblemente, la anciana recuerda seres queridos de su pasado). De manera azarosa y sin que el jugador pueda decidir nada, la anciana puede levantarse para dirigirse hacia la entrada del cementerio (es decir, volver por el camino inicial) o morir abruptamente, desplomándose en el banco y soltando el bastón.

El juego reduce la actividad del jugador hasta casi volverla innecesaria. No existe nada parecido a un objetivo o un desafío, por lo que The Graveyard, si bien asemeja visualmente a un videojuego, carece de dimensión lúdica y debe ser pensado desde otra perspectiva, quizás como una reflexión sobre la imposibilidad de actuar dentro del marco de un medio ergódico $y$, por lo tanto, como un texto que puede inscribirse dentro del campo todavía incierto del art game. The Graveyard circuló por los medios de prensa especializados en videojuegos y suele ser clasificado como "juego", por lo que su inclu- sión en este trabajo resulta pertinente, incluso si sus rasgos sugieren un funcionamiento discursivo diferente al lúdico.

La distancia respecto de las dos estrategias identificadas al principio es absoluta: aquí la muerte es el tema y la precariedad de la vejez se representa a través de la casi nula posibilidad de movimiento. A su vez, el caminar lento y dificultoso de la anciana, que se desplaza dando breves saltitos con su bastón, instaura un ritmo lento en el que el espacio adopta una temporalidad nueva, sobre todo si se lo compara con la velocidad con la que los juegos de géneros tradicionales conciben el desplazamiento de un lugar a otro. La canción acerca de personas fallecidas y enfermedades refuerza el tratamiento de la muerte como tema, y el fallecimiento de la anciana, totalmente azaroso, rompe decididamente con la concepción de la muerte asociada a una penalidad que se aplica ante la falta de competencias: aquí la muerte es contingente, incomprensible y ajena a cualquier clase de régimen lúdico. El morir no es un significante del fracaso, así como el juego no gestiona habilidades ni supone destrezas que permitan sortear la muerte.

\section{Conclusiones}

En este trabajo quise llamar la atención sobre tres cuestiones: los rasgos más o menos específicos que caracterizan a la muerte lúdica, y cómo esos rasgos alejan al videojuego de lo hecho por otros medios como el cine; las dos estrategias que parecen haber monopolizado la representación de la muerte hasta volverse convenciones cristalizadas en el espacio de diferentes géneros videolúdicos; señalar que esas estrategias son puestas en entredicho por juegos que ensayan diversas aperturas temáticas y lúdicas.

El carácter ergódico del medio habilita formas de tratamiento de la muerte que resultan inhallables en otros medios, por ejemplo, cuando el morir se 
transforma en una vía de aprendizaje y de adquisición de destrezas. Sin embargo, si bien el videojuego sedimentó un espectro representacional de la muerte, diversos productos del medio exhiben un movimiento contrario: el campo de lo verosímil, instaurado a partir de la repetición y de la restricción de los posibles, es horadado lentamente por juegos que ensayan distintas respuestas a un mismo problema estético presente en todos los medios y en la cultura en general: ¿cómo representar la muerte?

Los casos analizados tratan de responder a esa pregunta tomando diferentes grados de distancia del verosímil conformado por las dos estrategias mencionadas, ensayan diferentes formas de apertura respecto de esos límites estilísticos. Como se vio, el desvío puede ser mínimo, de orden más bien representativo. En la serie Karoshi la lógica del puzzle se articula con un objetivo inédito: ya no se trata de sobrevivir, sino de buscar activamente la muerte. En Everyday the Same Dream, en cambio, el morir no constituye un objetivo, aunque tampoco una penalidad, sino un elemento que instaura un clima de enrarecimiento y que habilita la tematización de una búsqueda personal, como si el morir se despegara del nivel lúdico y actuara en términos más plenamente semánticos. This War of Mine, por su parte, exhibe un abordaje del morir que imita al de la dramaturgia de otros géneros y lenguajes, donde la muerte modela tanto a los personajes como las relaciones que mantienen entre ellos y el entorno. Esto, que es perfectamente común en un medio como el cine, para el videojuego implica un desvío significativo: en This War of Mine el morir funciona en términos lúdicos, pero también lo hace dramáticamente a través de los efectos que produce la muerte tanto en los habitantes de la casa como en la economía precaria que el jugador debe administrar para no perder la partida. Por último, la muerte representada en The Graveyard implica un desenganche absoluto entre lo lúdico y el destino de la protagonista: las acciones disponibles se reducen a un mínimo, no hay despliegue posible de habilidades, peligros a sortear o problemas lógicos que descifrar (es decir, el juego no gestiona objetivos). En esa notoria caída de lo lúdico, la muerte de la anciana escapa completamente al desempeño del jugador y se transforma en una pura contingencia, algo que puede o no suceder con independencia de lo hecho durante el breve trayecto por el cementerio. En el espectro de desvío creciente que abren los casos elegidos, The Graveyard se ubica en el lugar más lejano: en el juego de Tale of Tales, el morir no está integrado al régimen lúdico (por ejemplo, sancionando la performance del jugador) y tampoco es representado de manera espectacular; el morir supone un acto azaroso y discreto, desconectado del desarrollo de la partida y carente de énfasis. Se trata, dentro del espectro representativo del videojuego, de una concepción trágica del morir, ya que la muerte no emerge como penalidad por un error ni es algo que pueda anticiparse o evitarse, sino que simplemente sucede, sin posibilidad de modificar el curso de los hechos. Si bien no se trata de la tragedia que Frasca vedada para el videojuego, The Graveyard sugiere que el medio puede tomar distancia de ciertas configuraciones del verosímil y ensayar nuevas modalidades expresivas que lo pongan en diálogo con lo hecho por otros medios y lenguajes.

Entonces, los casos elegidos revelan una expansión del campo de lo decible del videojuego: con el paso de los años, el medio encuentra soluciones estéticas nuevas para el viejo problema de la representación de la muerte y ensanchar sus posibilidades expresivas. El análisis de los casos arroja un panorama rico en matices donde la tensión entre juego y narración se resuelve de diferentes maneras a través de configuraciones textuales originales que proponen nuevas formas de contacto discursivo. 


\section{Referencias bibliográficas}

Aarseth, E. (1997). Cybertext: Perspectives on ergodic literature. Baltimore, Estados Unidos: John Hopkins University Press.

Adams, M. (2014). Spectacular mortality: Intersections of punitive \& educational player death. First Person Scholar. Recuperado de http://www. firstpersonscholar.com/spectacular-mortality

Barthes, R. (1972). Introducción al análisis estructural de los relatos. En R. Barthes. (Ed), Análisis estructural del relato (pp 7-38). Buenos Aires, Argentina: Tiempo Contemporáneo.

Burch, N. (2011). El tragaluz del infinito: Contribución a la genalogía del lenguaje cinematográfico. Madrid, España: Cátedra.

Frasca, G. (2003). Ludologists love stories, too: Notes from a debate that never took place. En J. Raessens, M. Copier, J. Goldstein, F. Mäyrä (Presidencia), DiGRA 2003. Level Up. Utrecht, Países Bajos.

Gorvett, Z. (09 de octubre de 2016). Qué es el "karoshi", la muerte por exceso de trabajo que en Japón es un problema de salud pública. $B B C$. Recuperado de http://www.bbc.com/mundo/ vert-cap-37391172

Klevjer, R. (2002). In defense of cutscenes. En M. Huotari (Presidencia), Computer Games and Digital Cultures Conference. Tampere, Finlandia.

Harrer, S. (2013). From losing to loss: Exploring the expressive capacities of videogames beyond death as failure. Culture Unbound, 5, 607-620.

Maté, D. (2016) Representaciones de la muerte en el videojuego. Lo lúdico y lo narrativo entre la norma y el desvío. Lexia. Rivista di semiotica, 21(22), 285-300.

Metz, C. (1975). El decir y lo dicho en cine. En: R. Barthes, M.C. Boons, O. Burgelin, G. Genette, J. Gritti, J. Kristeva, C. Metz, V. Morin y T. Todorov. (Eds.), Lo verosímil (pp. 19-30). Buenos Aires, Argentina: Tiempo Contemporáneo.

Metz, C. (1991). Lenonciation impersonelle ou le site du filme. París, Francia: Klincksieck.

Mukehrjee, S. (2009). Remembering how you died: Memory, death and temporality in videogames. En T. Krzywinska, H. Kennedy, B. Atkins (Presidencia), DiGRA 2009. Breaking New Ground: Innovation in Games, Play, Practice and Theory. Londres, Gran Bretaña.

Neto, M. (2015). A hero's death: Human mortality and video games. Santa Cruz: University of California. Recuperado de https://www.academia. edu/10622358/A_Heros_Death_Human _ Mortality_and_Video_Games?auto=download

Segre, C. (1988) Principios de análisis del texto literario. Barcelona, España: Crítica.

Steimberg, O. (2013) Semióticas: las semióticas de los géneros, de los estilos, de la transposición. Buenos Aires, Argentina: Eterna Cadencia.

Tocci, J. (2008). You are dead. Continue?: Conflicts and complements in game rules and fiction. Eludamos. Journal for Computer Game Culture, 2(2), 187-201.

Verón, E. (1996). La semiosis social: fragmentos de una teoría de la discursividad. Barcelona, España: Gedisa. 